\title{
Microbial functional change is linked with clinical outcomes after capsular fecal transplant in cirrhosis
}

Jasmohan S. Bajaj, ${ }^{1}$ Nita Salzman, ${ }^{2}$ Chathur Acharya, ${ }^{1}$ Hajime Takei, ${ }^{3}$ Genta Kakiyama, ${ }^{1}$ Andrew Fagan, ${ }^{1}$ Melanie B. White, ${ }^{1}$ Edith A. Gavis, ${ }^{1}$ Mary L. Holtz, ${ }^{2}$ Michael Hayward, ${ }^{2}$ Hiroshi Nittono, ${ }^{3}$ Phillip B. Hylemon, ${ }^{4}$ I. Jane Cox, ${ }^{5}$ Roger Williams, ${ }^{5}$ Simon D. Taylor-Robinson, ${ }^{6}$ Richard K. Sterling, ${ }^{1}$ Scott C. Matherly, ${ }^{1}$ Michael Fuchs, ${ }^{1}$ Hannah Lee, ${ }^{1}$ Puneet Puri, ${ }^{1}$ R. Todd Stravitz, ${ }^{1}$ Arun J. Sanyal, ${ }^{1}$ Lola Ajayi, ${ }^{5}$ Adrien Le Guennec, ${ }^{7}$ R. Andrew Atkinson, ${ }^{7}$ Mohammad S. Siddiqui, ${ }^{1}$ Velimir Luketic, ${ }^{1}$ William M. Pandak, ${ }^{1}$ Masoumeh Sikaroodi, ${ }^{8}$ and Patrick M. Gillevet ${ }^{8}$

'Division of Gastroenterology, Hepatology and Nutrition, Virginia Commonwealth University and McGuire VA Medical Center Richmond, Virginia, USA. ²Department of Pediatrics, Medical College of Wisconsin, Milwaukee, Wisconsin, USA. ${ }^{3}$ unshin Clinic Bile Acid Institute, Meguro-Ku, Tokyo, Japan. ${ }^{4}$ Department of Microbiology and Immunology, Virginia Commonwealth University and McGuire VA Medical Center, Richmond, Virginia, USA. ${ }^{5}$ Institute for Hepatology London, Foundation for Liver Research, London, United Kingdom. ${ }^{6}$ Department of Surgery and Cancer, Imperial College London, London, United Kingdom. ${ }^{7}$ Randall Centre for Cell \& Molecular Biophysics and Centre for Biomolecular Spectroscopy, King's College London, London, United Kingdom. ${ }^{8}$ Microbiome Analysis Center, George Mason University, Manassas, Virginia, USA.

BACKGROUND. Hepatic encephalopathy (HE) is associated with poor outcomes. A prior randomized, pilot trial demonstrated safety after oral capsular fecal microbial transplant (FMT) in HE, with favorable changes in microbial composition and cognition. However, microbial functional changes are unclear. The aim of this study was to determine the effect of FMT on the gut-brain axis compared with placebo, using microbial function based on bile acids (BAs), inflammation (serum IL-6, LPS-binding protein [LBP]), and their association with EncephalApp.

METHODS. Twenty cirrhotic patients were randomized 1:1 into groups that received 1-time FMT capsules from a donor enriched in Lachnospiraceae and Ruminococcaceae or placebo capsules, with 5-month follow-up for safety outcomes. Stool microbiota and BA; serum IL-6, BA, and LBP; and EncephalApp were analyzed at baseline and 4 weeks after FMT/placebo. Correlation networks among microbiota, BAs, EncephalApp, IL-6, and LBP were performed before/after FMT.

RESULTS. FMT-assigned participants had $1 \mathrm{HE}$ recurrence and 2 unrelated infections. Six placeboassigned participants developed negative outcomes. FMT, but not placebo, was associated with reduced serum IL- 6 and LBP and improved EncephalApp. FMT-assigned participants demonstrated higher deconjugation and secondary BA formation in feces and serum compared with baseline. No change was seen in placebo. Correlation networks showed greater complexity after FMT compared with baseline. Beneficial taxa, such as Ruminococcaceae, Verrucomicrobiaceae, and Lachnospiraceae, were correlated with cognitive improvement and decrease in inflammation after FMT. Fecal/serum secondary/primary ratios and PiCRUST secondary BA pathways did not increase in participants who developed poor outcomes.

declared that no conflict of interest exists.

Copyright: (c) 2019, American Society for Clinical Investigation.

Submitted: September 11, 2019 Accepted: November 13, 2019 Published: November 21, 2019.

Reference information: /CI Insight. 2019;4(24):e133410. https://doi.org/10.1172/jici. insight.133410.

CONCLUSION. Gut microbial function in cirrhosis is beneficially affected by capsular FMT, with improved inflammation and cognition. Lower secondary BAs in FMT recipients could select for participants who develop negative outcomes.

TRIAL RECISTRATION. Clinicaltrials.gov NCT03152188.

FUNDING. National Center for Advancing Translational Sciences NIH grant R21TR002024, VA Merit Review grant 2I0CX001076, the United Kingdom National Institute for Health Research Biomedical Facility at Imperial College London, the British Heart Foundation, Wellcome Trust, and King's College London. 


\section{Introduction}

An altered gut-liver-brain axis underlies the pathogenesis of hepatic encephalopathy (HE) $(1,2)$. Therefore, beneficial alterations of the microbiota composition and function in HE are usually the mainstay of therapy (1). Treatments using laxatives, such as lactulose, and nonabsorbable antibiotics, such as rifaximin, are traditionally used; however, other options are required in patients not responding to such therapies (1). Fecal microbial transplant (FMT), using the oral capsular and enema route has the capability of changing the microbial milieu in several diseases $(3,4)$. In a prior randomized trial of patients with HE already on rifaximin and lactulose, FMT capsules were associated with engraftment of donor microbiota into the stool and mucosa, which resulted in improved cognitive function, as found using OffTime+OnTime in the EncephalApp, and decrease in overall hospitalizations compared with placebo $(3,4)$. In this study, we found that 3 FMT-assigned patients either had an infection or were hospitalized, while 6 placebo-assigned patients experienced similar complications. There was also a reduction in serum LPS-binding protein (LBP) in FMT but not in placebo patients.

While there was compositional improvement, interaction of inflammation, bacterial translocation, and microbial function (bile acid [BA]) with cognition need to be evaluated (4). Gut microbiota can transform BAs by deconjugation, converting primary BAs to secondary and tertiary (oxo-, sulfated, urso-, and iso-BA) forms, which have important functional consequences (5).

Our aim was to determine the linkage between changes in microbiota after FMT with changes in serum and fecal BA moieties, inflammation, untargeted metabolic profiling of urine and serum, and cognition in patients with cirrhosis and HE compared with placebo.

\section{Results}

We enrolled 20 patients who were matched with respect to demographics, cirrhosis severity, and cognitive testing (Supplemental Table 2 and Supplemental Figure 1; supplemental material available online with this article; https://doi.org/10.1172/jci.insight.133410DS1). All patients were on lactulose and rifaximin, and the clinical course has been characterized in Supplemental Table 3: 6 patients in the placebo group and 1 person in the FMT group experienced serious adverse events. There were also 3 patients in the placebo group and 2 in the FMT group with infections, which were considered unrelated by the Data Safety Monitoring Board. None of the clinical laboratory data significantly changed over time between and within groups (Supplemental Table 4).

\section{Microbiota analysis}

As published previously, there was an increase in relative abundance of Lachnospiraceae and Ruminococcaceae in the FMT group after FMT compared with baseline, while there were no significant changes in the stool of the placebo group (4). There was also an increase in relative abundance of these taxa in the duodenal and sigmoid mucosa in the FMT group, indicating satisfactory engraftment.

\section{Cognitive change}

As published previously, there was a significant improvement, i.e., reduction in EncephalApp OffTime+OnTime values in the FMT group (before $277.8 \pm 123.5$ vs. after $226.7 \pm 56.1$ seconds, $P=0.04$ paired $t$ test) but not in placebo group (before $318.9 \pm 181.0$ vs. after $308.9 \pm 169.5, P=0.47$ ) (4).

\section{Inflammation}

After FMT, but not placebo, there was a significant reduction in serum IL-6 (Figure 1B) (4).

\section{Metabolomic data}

Excellent quality NMR data were obtained from all the serum and urine samples. However, neither the serum nor the urine NMR data sets showed differences before FMT/after FMT, before placebo/after placebo, or after FMT/after placebo using multivariate analyses techniques (principal component analysis, class separation 0.00, and partial least-squares discriminant analysis, $\mathrm{Q} 2<0.25$ ). Detailed results are in the Supplemental Methods and Supplemental Figure 2.

\section{BA analysis}

Fecal and serum BA. We focused on 3 levels of microbial action on fecal BAs: deconjugation, 7 $\alpha$-dehydroxylation, and formation of tertiary BAs. At baseline, there were no significant differences in the concentrations of fecal 


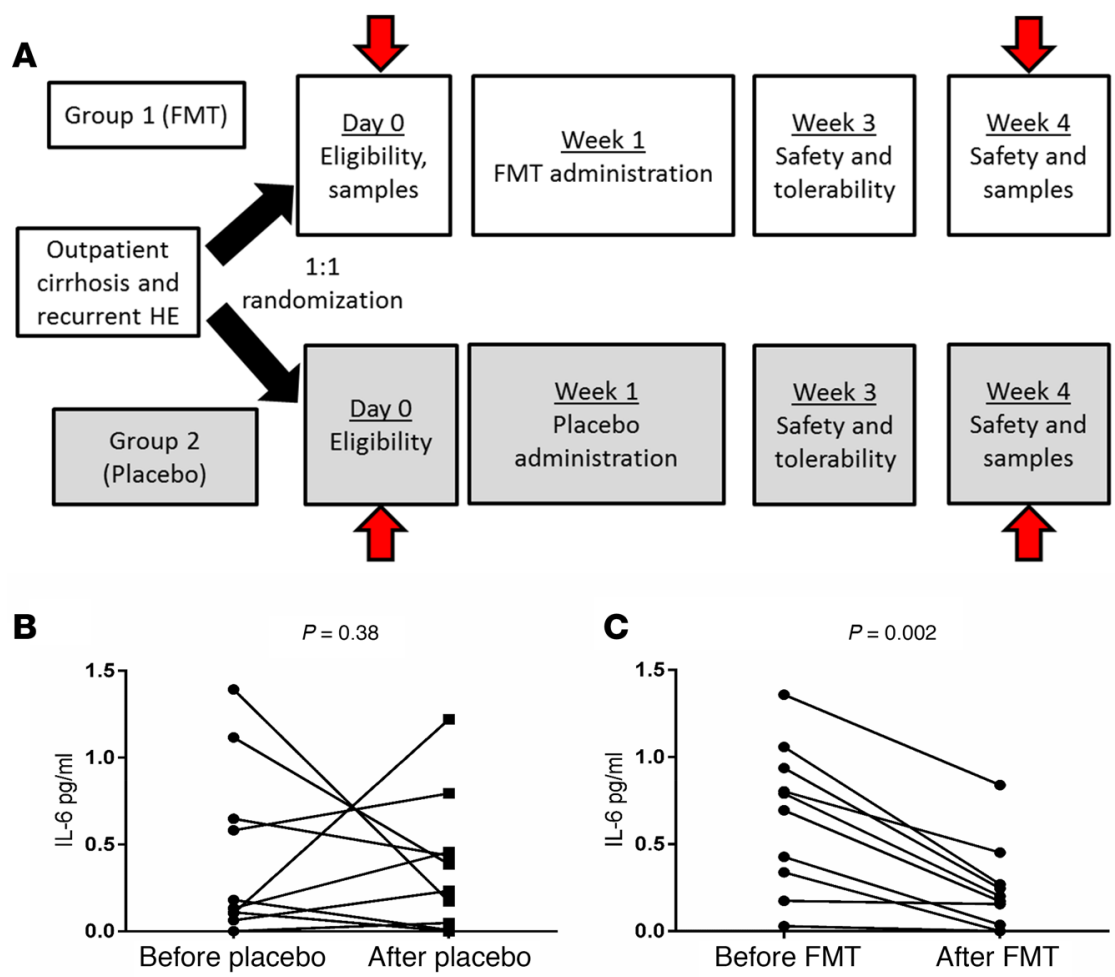

Figure 1. Schema of Study and Changes in serum IL-6 values. (A) Schema of the initial 4 weeks, with arrows indicating when the stool, urine, and serum were collected and analyzed. Clinical follow-up was for a 5-month period afterward. (B) Serum IL-6 $(\mathrm{pg} / \mathrm{mL})$ change within the placebo group was not significant. (C) Serum IL-6 in the FMT group showed significant reduction compared with baseline. $P$ values on Wilcoxon matched-pairs tests are shown. FMT, fecal microbiota transplant.

BAs (Table 1) as well the relative proportion of primary, secondary, and tertiary BAs between placebo and FMT groups. Four weeks after FMT capsules, there was a statistically similar total bile acids between and within groups. After FMT, there was an increase in deconjugation, as shown by a lower conjugated BA concentration. This was accompanied by a marked reduction in total primary BAs and an increase in secondary BAs and secondary/primary BA ratio after FMT (Figure 2, A and B; and Figure 3, A and B). There was a reduction prominent in cholic acid, but both deoxycholic and lithocholic acid concentrations increased after FMT. No significant change in fecal tertiary BAs (iso, oxo, or sulfated) were seen. This was accompanied by a similar pattern in serum, with lower primary and higher secondary BAs in the FMT group but not in the placebo group (Table 2 and Figure 3, C and D). Conjugation status did not change, and both glyco- and tauro-conjugated primary BA moieties (chenodeoxycholic and cholic) reduced after FMT. No changes in total BAs were seen in the serum nor were changes in the tertiary or conjugated BAs seen in the post-FMT group compared with placebo groups.

PiCRUST results. Focusing on predicted functionality for secondary BA synthesis, we found a significantly higher expression after FMT compared with baseline, while a trend toward lower expression was seen in the placebo group over the same period (Figure 4).

\section{Correlation network analysis}

There was a significant increase in cumulative distribution frequency of the correlations between microbiota, EncephalApp, BAs, IL-6, and LBP after FMT compared with the pre-FMT state (Figure 5A). The actual network differences are shown in Figure 5B. A high score on EncephalApp indicates poor performance. After FMT, Verrucomicrobiaceae were negatively associated with EncephalApp and IL-6, and Verrucomicrobiaceae were positively associated with Ruminococcaceae. Baseline positive correlation of Enterococcaceae with EncephalApp and LBP disappeared after FMT. IL-6 showed new negative correlations with Clostridiales Incertae Sedis XIV after FMT. Baseline negative Saccharibacteria correlations with EncephalApp and Lachnospiraceae were not seen after FMT. Fecal BA changes included positive linkage of conjugated deoxycholic acid with IL-6 and negative with EncephalApp at baseline, which disappeared after FMT. 
Table 1. Stool bile acid concentrations

\begin{tabular}{|c|c|c|c|c|}
\hline \multirow{2}{*}{$\begin{array}{c}\text { Median } \\
\text { ( } \mu \mathrm{g} / \mathrm{gm} \text { stool) }\end{array}$} & \multicolumn{2}{|c|}{ Placebo } & \multicolumn{2}{|c|}{ Fecal microbial transplant } \\
\hline & Baseline & End & Baseline & End \\
\hline Total BAs & $2.67(4.38)$ & $2.6(8.26)$ & $5.66(8.87)$ & $6.3(10.72)$ \\
\hline Total conjugated & $0.39(0.89)$ & $0.29(0.55)$ & $0.72(3.94)$ & $0.07(0.13)^{A}$ \\
\hline Total primary & $1.65(1.35)$ & $1.46(2.41)$ & $1.54(8.01)$ & $1.10(2.39)^{\mathrm{A}}$ \\
\hline Cholic acid & $0.38(1.0)$ & $0.21(1.04)$ & $0.61(3.64)$ & $0.04(0.90)^{A, B}$ \\
\hline Chenodeoxycholic acid & $0.38(0.75)$ & $0.27(0.43)$ & $0.25(2.28)$ & $0.10(0.26)$ \\
\hline Lithocholic acid & $0.18(1.13)$ & $0.10(0.95)$ & $0.51(1.29)$ & $1.11(2.78)^{A, B}$ \\
\hline Secondary/primary BA & $0.18(7.03)$ & $0.53(5.40)$ & $4.49(9.27)$ & $9.83(12.72)^{A, B}$ \\
\hline Total tertiary & $0.62(1.47)$ & $0.90(2.55)$ & $1.24(2.81)$ & $1.27(2.49)$ \\
\hline Total oxo-BAs & $0.26(0.50)$ & $0.28(0.62)$ & $0.46(0.91)$ & $0.46(0.43)$ \\
\hline Total iso-BAs & $0.10(0.57)$ & $0.10(1.01)$ & $0.27(0.72)$ & $0.48(0.93)$ \\
\hline
\end{tabular}

Data are presented as median (IQR). ${ }^{A} P<0.05$, Wilcoxon signed-rank matched-pairs tests, ${ }^{B} P<0.05$ between groups, Mann-Whitney test. BA, bile acid.

\section{Association of microbial functional changes on outcomes}

Four FMT-assigned and seven placebo-assigned patients had low secondary/primary BA ratios in serum and feces at baseline. Of these, 3 FMT-assigned patients continued to not have an increase in the secondary/primary BA ratios compared with baseline, even after FMT, in the serum or feces, while in placebo group, this was seen in all 6 patients (Figure 3, A-D). This corresponded to the PiCRUST (Phylogenetic Investigation of Communities by Reconstruction of Unobserved States) secondary BA synthesis genes.

Of the 3, 1 was admitted with HE within 30 days, and 2 had subsequent infections and were treated as outpatients. All 6 of the placebo group patients, who did not have increased secondary BAs, developed HE or infections during the follow-up. Therefore, all 9 patients who developed outcomes during the follow-up did not demonstrate appreciable fecal or serum secondary/primary BA ratios. None of the other BA moieties, IL-6, LBP, or untargeted metabolomics were associated with outcomes.

\section{Discussion}

The data demonstrate that after oral capsular FMT performed as a randomized, placebo-controlled trial, there are distinct microbial functional changes in the FMT-assigned group compared with the placebo-assigned group. Improvements in cognitive function were linked with changes in Verrucomicrobiaceae, IL-6, and Ruminococcaceae in oral capsular FMT. Microbial transformation of BAs was associated with outcomes and may be relevant to monitor the success of the FMT.

Microbial perturbations are associated with the pathogenesis, and prognostication in $\mathrm{HE}$ and most therapies are focused on improving these alterations $(1,6)$. However, a major subset of patients continues to suffer cognitively and clinically despite the standard of care, which is lactulose and rifaximin in the US (7). The prevention of further deterioration is important in these patients. Two prior randomized clinical phase I trials have shown safety of the enema and oral capsular route in FMT in cirrhosis and recurrent HE compared with the comparator group $(3,4)$. The enema trial, performed after/before FMT broad-spectrum antibiotics, was associated with improved cognition and lowered hospitalizations (3). However, functional changes in BA physiology, shortchain fatty acids, and untargeted metabolomics in this trial largely demonstrated a restoration of preantibiotic baseline (8). In the cirrhosis population with limited ability to restore diversity, this is relevant, but questions regarding the role of FMT itself in altering microbial function in cirrhosis are still open.

Therefore, the analysis of microbial functional change after FMT in the oral capsular FMT trial, which was done without postintervention broad-spectrum antibiotics, was performed. We found changes in several aspects of microbial function and inflammation as a result of FMT in this study, which demonstrates a potential beneficial effect. There was a significant alteration in the bacterial biotransformation of BAs, which spanned both fecal and serum compartments. After oral capsular FMT, there was higher evidence of deconjugation and $7 \alpha$-dehydoxylation of bile acids compared with 
A

Fecal BA change in the FMT group

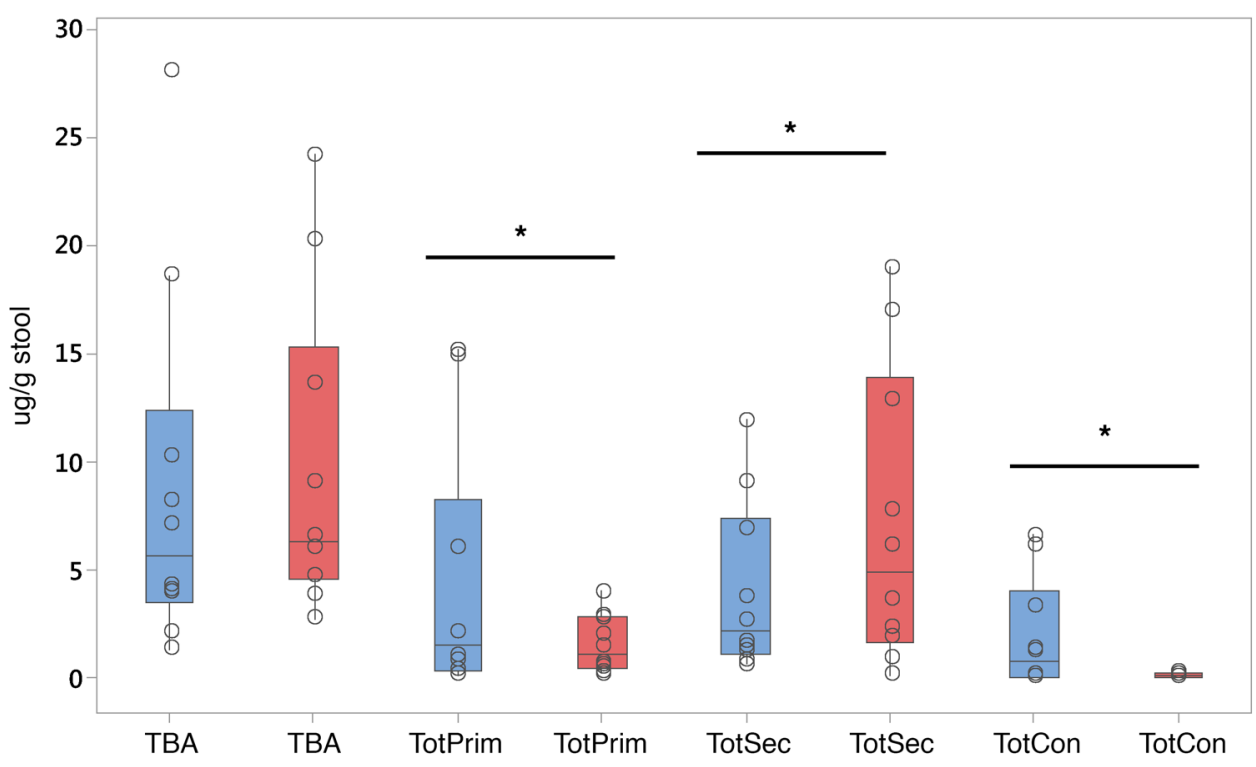

Figure 2. Bile acid concentrations. Data are presented as median and $95 \% \mathrm{Cl}$ and individual values. Within group comparisons were performed using Wilcoxon matched-pairs test, while Mann Whitney was used for between group analyses. Blue columns represent baseline values, while red columns represent 4-week postintervention values. (A) There was a significant reduction in conjugated and primary bile acids (BAs) and an increase in secondary BAs after FMT compared with baseline. (B) No significant change in these moieties was seen in the placebo group. ${ }^{*} P<0.05$ on Wilcoxon matched-pairs test as appropriate. Data are presented as $\mu / g$ of dried stool.

B

Fecal BA change in the placebo group

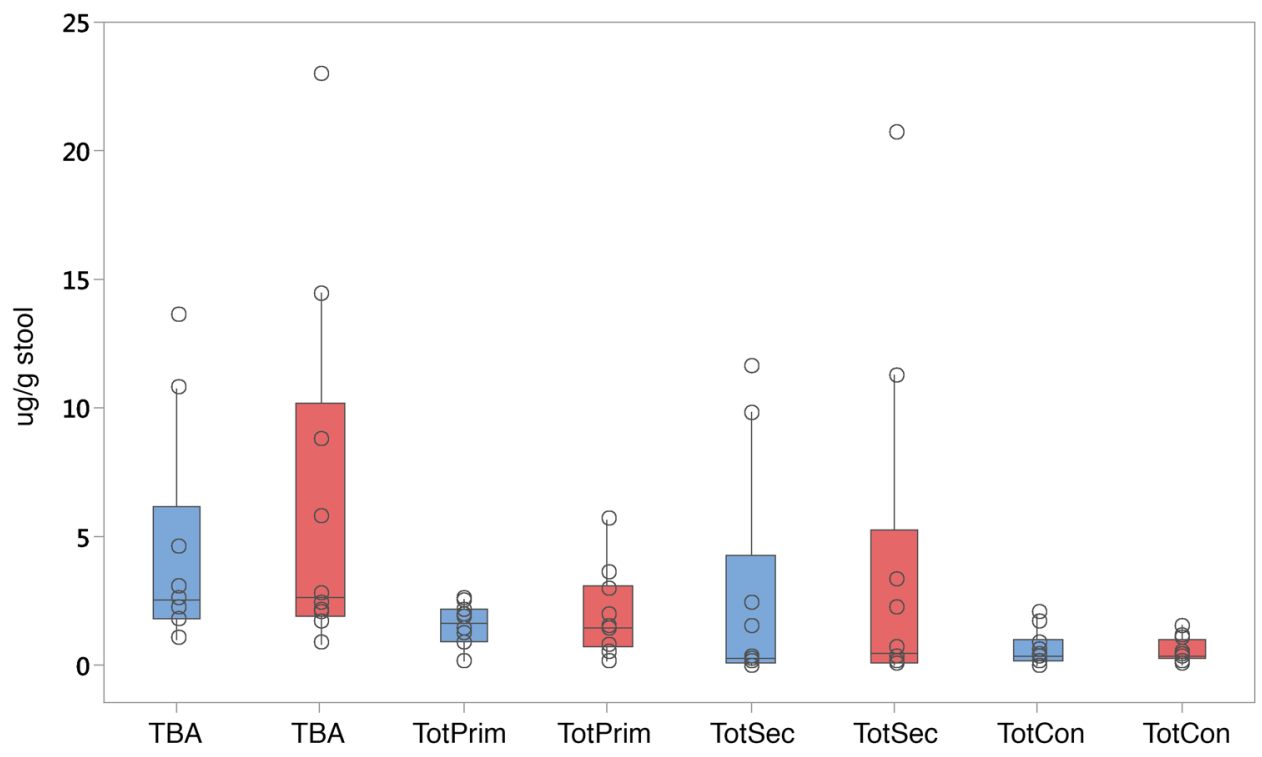

baseline and with the placebo group, which resulted in higher secondary/primary fecal and serum BA ratios. No changes in UDCA or tertiary BA moieties were seen. Deconjugation is the first step before 7 $\alpha$-dehydoxylation; it is usually not impaired in decompensated cirrhosis (5). On the other hand, patients with decompensated cirrhosis typically are not able to produce secondary BAs due to the relative reduction in Clostridial spp $(5,9)$. The microbiota in the donor was enriched in these taxa and likely resulted in the increases in secondary BA. These findings were also corroborated by the predicted functionality assay using PiCRUST. This particular set of changes demonstrates a step toward return to healthy microbial function, which has also been seen after successful FMT in patients with C. difficile (10). Indeed, all 9 subjects who had adverse events, such as recurrent $\mathrm{HE}$ and infections, had a continued lack of increase in secondary/primary BA ratios in the serum and feces. These included 3 FMT patients whose secondary BA profiles and whose corresponding PiCRUST-predicted genes remained stagnant compared with those before FMT. Secondary BAs are associated with protection from pathogenic organisms, indicate a healthy functional microbial diversity, and could also indicate 
Table 2. Serum bile acid concentrations

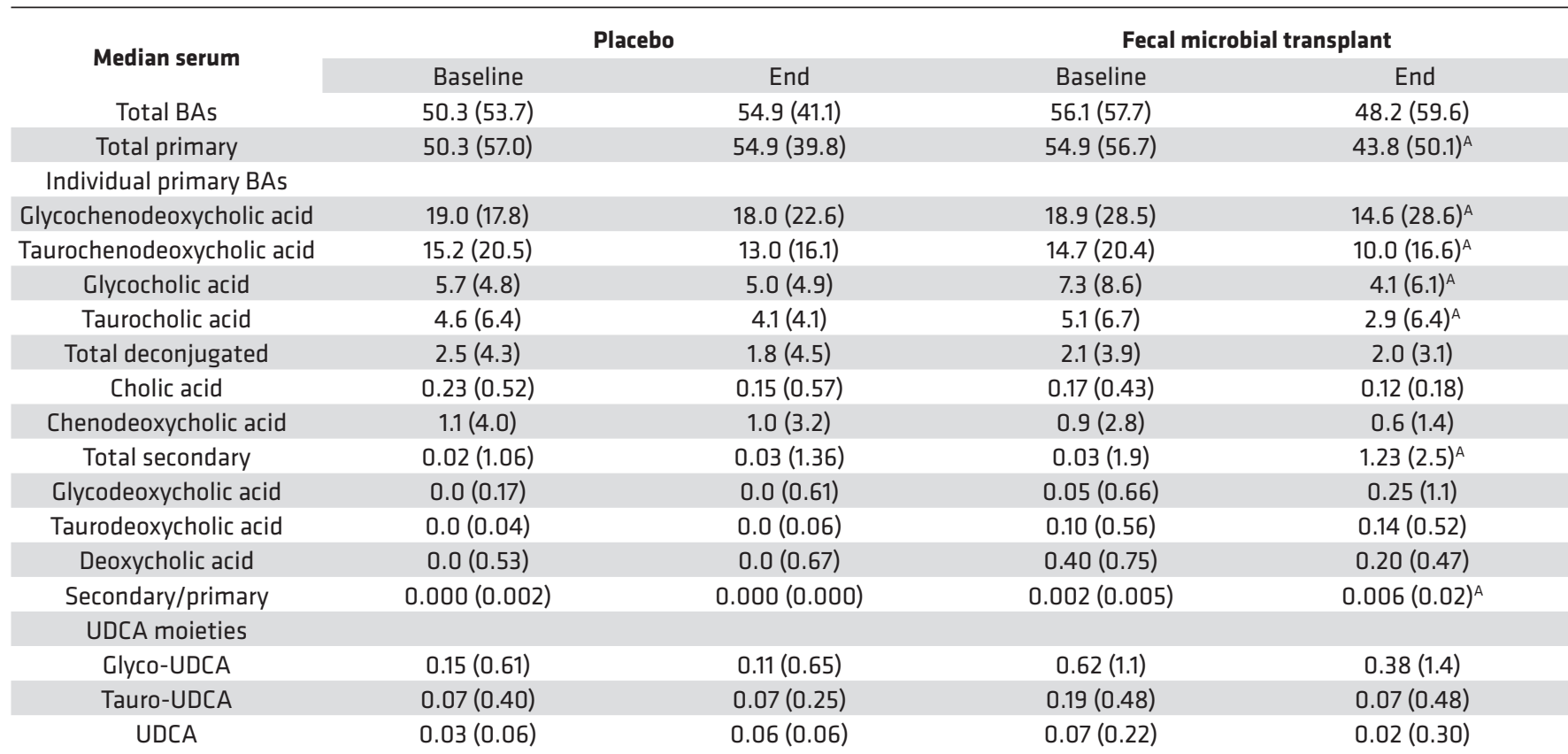

Data are presented as median (IQR). ${ }^{A} P<0.05$ Wilcoxon signed rank matched-pairs tests. BA, bile acid; UDCA, ursodeoxycholic acid.

presence of taxa that make other compounds to reduce pathogenic microbiota $(11,12)$. The changes in BA biotransformation in cirrhosis could have prognostic significance and could be a tool to ensure engraftment from a functional, rather than simply a compositional perspective.

The immunoinflammatory milieu is also an important pathogenetic determinant of outcomes and cognitive impairment in cirrhosis (13). We found a reduction in serum LBP and IL-6 after FMT but not after placebo, which demonstrates improvement in this milieu. Moreover, correlation network analyses showed a change in complexity and that beneficial microbial taxa, such as Ruminococcaceae and Verrucomicrobiaceae, were linked with the improved immunoinflammatory milieu and with EncephalApp performance. Verrucomicrobiaceae, which includes Akkermansia muciniphila, are associated with decreased inflammation and strengthened intestinal barrier in patients with and without liver disease (14-17). In addition, the presence of these taxa was associated with lowered neuroinflammation in mice colonized after FMT from humans with cirrhosis (14). We chose the human donor enriched in Ruminococcaceae, which in turn has taxa that can dehydroxylate primary to secondary BAs and were associated with lowered IL-6 after FMT. These correlations point toward a beneficial shift in the immunoinflammatory milieu systemically and locally due to FMT that could result in improvement in cognitive performance.

It is intriguing that untargeted metabolomics of the serum and urine did not reflect changes overall in microbial or other metabolites either within or between groups. This contrasted with findings from a prior enema FMT trial, in which the urinary metabolite changes observed after both FMT and antibiotics returned to baseline after FMT (3). These differences could be due to the major changes influenced by broad-spectrum antibiotics that were likely not reflected by the capsular FMT. It is also likely that several changes brought on by FMT alone were more at the intestinal interface and focused on bacterial products, such as LBP and BAs, rather than an overall change in metabolic milieu. Therefore, a more focused approach to metabolomics, rather than an untargeted one or one using fecal metabolomics, could be important to tease out results based on FMT without antibiotics.

Our study is limited by the relatively small number of patients who received oral capsular FMT and the even smaller number who developed infections or HE. Therefore, larger sample sizes are needed to further evaluate whether changes in BA profiles can predict outcomes definitively. All our subjects, regardless of oral capsular FMT or placebo grouping, were on rifaximin and PPI, which can affect microbial function and could affect engraftment $(18,19)$. However, these therapies remained constant throughout the study and rifaximin is 
A
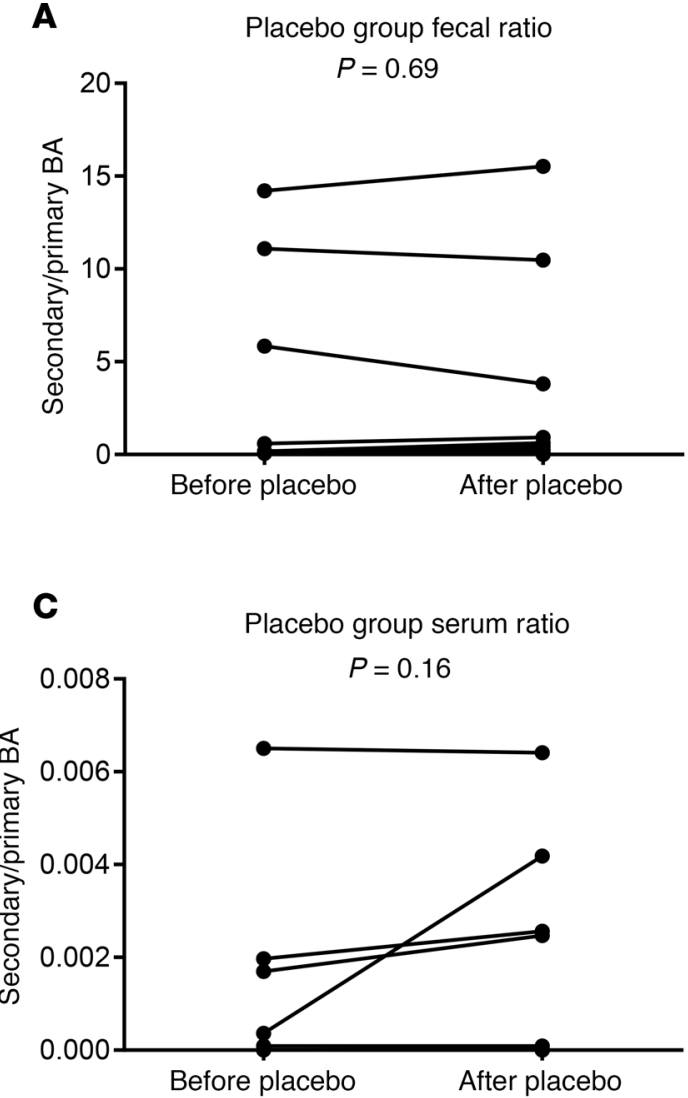

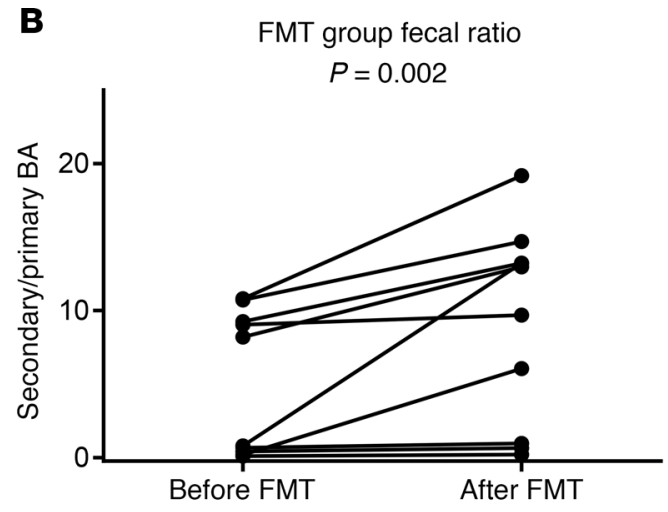

D

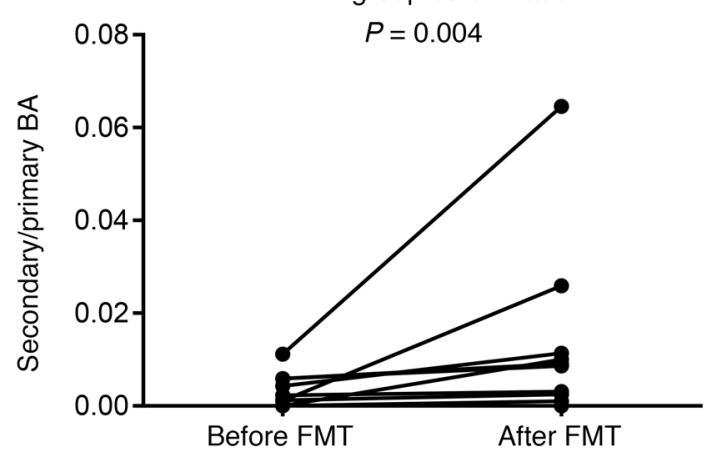

Figure 3. Secondary/primary bile acid ratios. (A and B) Stool secondary/primary BA ratio. Individual values showing similar values in placebo (A) and increase in all but 3 in FMT (B). (C and D) Serum secondary/primary BA ratio. Individual values showing similar values in placebo (C) and increase in all but 3 in FMT (D). All comparisons were done using Wilcoxon matched-pairs test as appropriate and $P$ values of comparisons are shown.

associated with an increase in fecal secondary BA concentrations (9). We also performed microbial functional analyses at week 4 rather than at the end of the study. However, engraftment and resultant bacterial function changes are more likely closer to the FMT results at the 4-week point rather than later results. Future studies are needed in patients with cirrhosis not on proton pump inhibitors, lactulose, or rifaximin. Given the recent reports of infection transmission with FMT from donors that were not checked with updated strict protocols, this remains an investigational therapy, and further research is needed (20). The donor in this study was negative for ESBL-producing E. coli and other known pathogens per the strict requirements of Openbiome (21).

We conclude that gut microbial function is beneficially affected by capsular FMT, with amelioration of the systemic inflammatory milieu and cognitive improvement in cirrhosis and recurrent HE. The absence of secondary BAs in FMT recipients could select for participants who develop poor outcomes on follow-up. Larger studies are needed to determine the ability of early microbial functional change to prognosticate longer-term outcomes after FMT in cirrhosis and HE.

\section{Methods}

\section{Randomized placebo-controlled clinical trial}

As previously published, we enrolled patients with cirrhosis with recurrent $\mathrm{HE}$ already on lactulose and rifaximin, all of whom were also on proton pump inhibitors, into a clinical trial under an FDA IND (Figure 1A) (Clinicaltrials.gov NCT03152188) (4). Patients were randomized 1:1 into a group receiving 15 FMT capsules all at one occasion and a group receiving identical placebo. FMT was from a single donor enriched in Lachnospiraceae and Ruminococcaceae, selected through machine learning. Details regarding the trial design and eligibility criteria and methods are in the Supplemental Methods and Supplemental Table 1 . We 

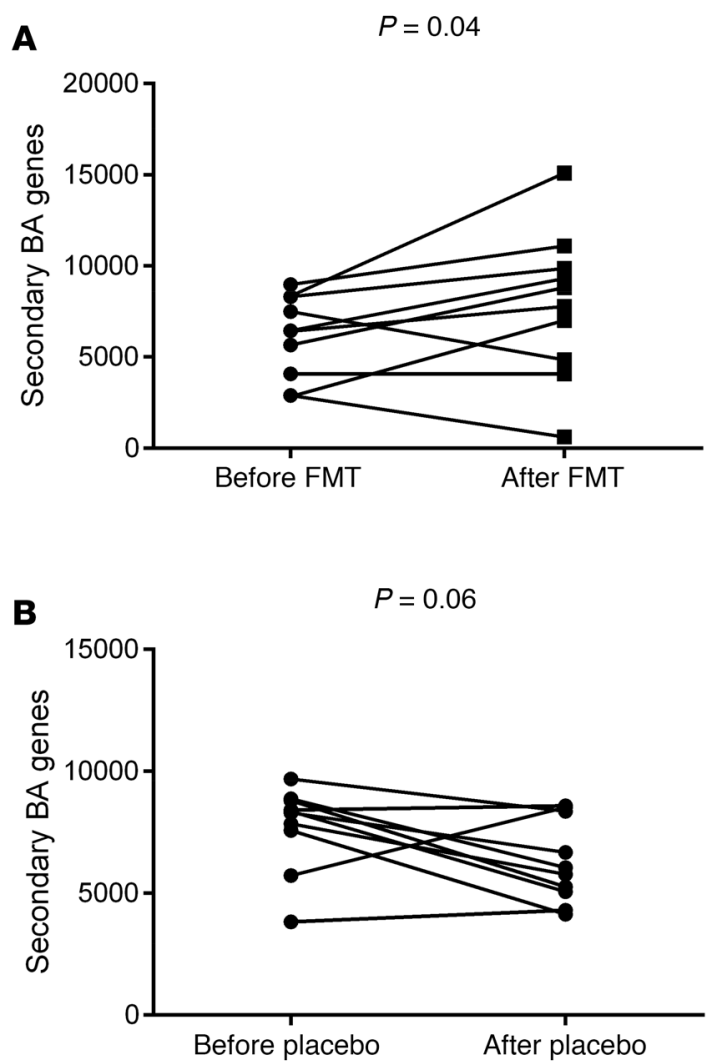

Figure 4. PiCRUST changes in the secondary bile acid synthesis genes before versus after intervention. (A) Individual values of secondary bile acid synthesis genes before versus after FMT show a significant increase, with 3 patients' values either similar or lower. These were the same as those whose serum and fecal values were low after FMT. (B) Individual values of secondary bile acid synthesis genes before versus after placebo show a trend toward decrease over baseline.

administered 15 capsules of FMT versus placebo once to each participant. The 15 capsules contained 4.125 grams of stool, and they were created using wet preparation of stool without lyophilization.

\section{Endpoints of the trial}

Hospitalizations and infections, serious adverse events related to FMT, cognitive function using the psychometric HE score and EncephalApp Stroop, and the microbial composition at the mucosal and fecal levels were analyzed (4). These were compared between and within groups at baseline and 4 weeks after intervention, and subjects were followed for 5 months. All groups provided serum, urine, and stool at the screening visit, study baseline visit, safety visit (1-2 weeks after placebo or FMT capsules), and at 4 weeks after placebo or FMT capsule.

\section{Microbial composition and function}

Microbial composition. We used published techniques to evaluate 16SrRNA sequencing from stool and mucosal biopsies from the sigmoid and duodenum. Diversity and relative abundances were compared between and within groups using Shannon indices and QIIME2 (quantitative insight into microbial ecology).

\section{Microbial function}

$B A$ analysis. Serum and fecal BA analysis were performed using published LC/MS techniques (22). Details regarding specific moieties that were determined can be found in the Supplemental Methods. We determined the total BA, primary BA, secondary BA, conjugated BA, and tertiary BA at baseline and study end and within groups. Tertiary BAs included ursodeoxycholic acid (UDCA) and oxo and iso forms of BAs. Secondary/primary fecal BA ratios were also calculated.

PiCRUST (23) was performed focusing on the secondary BA synthesis pathway in patients before/after FMT and before/after placebo with respect to stool microbiota.

Untargeted metabolomics. Urine and serum samples were prepared for NMR analyses using standardized protocols (24). NMR data were acquired at the Centre for Biomolecular Spectroscopy, King's College London, using a Bruker 600-MHz (AVANCE NEO) NMR spectrometer with a ${ }^{1} \mathrm{H} /{ }^{13} \mathrm{C} /{ }^{15} \mathrm{~N}$ TCI Prodigy (nitrogen-cooled) probe (Bruker). Pulse-collect (urine, serum) and spin-echo (serum) NMR data sets were 
A

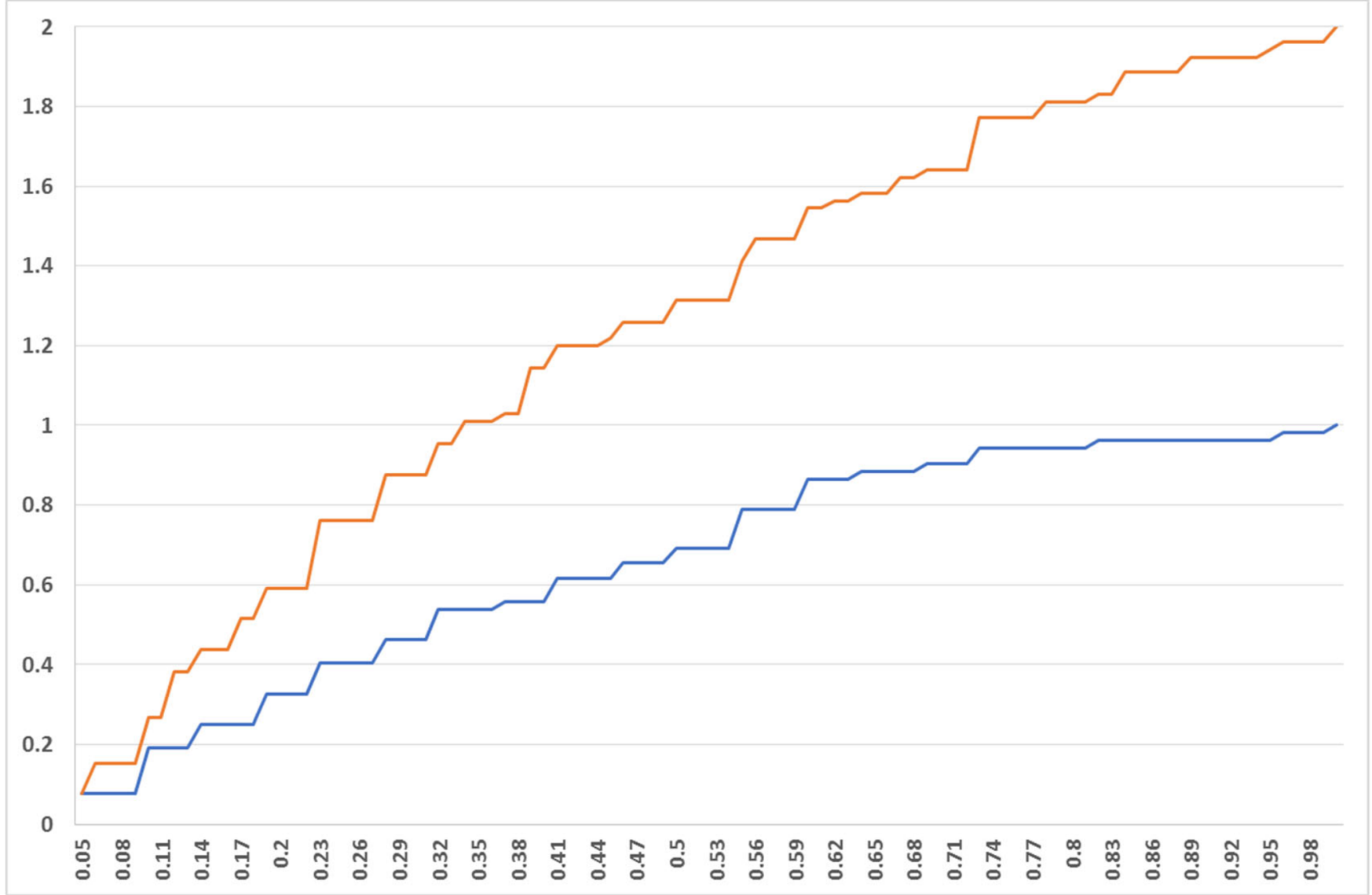

B

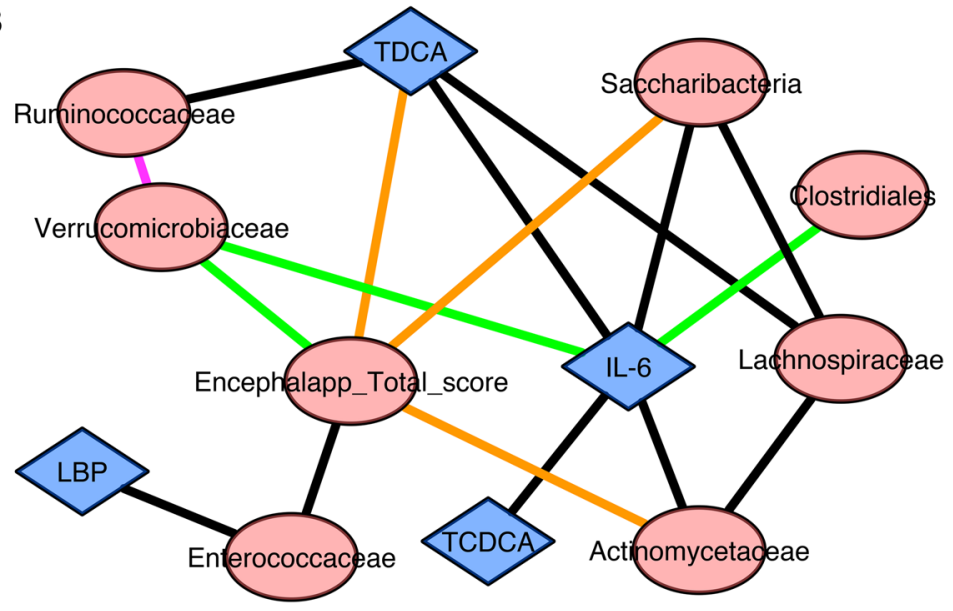

Figure 5. Correlation networks and difference. (A) Cumulative distribution frequency after FMT was higher than that before FMT in the correlation networks. (B) Pre- versus post-FMT correlation network differences showed changes centered around EncephalApp and serum IL-6. Purple line: positive after FMT: none at baseline; green lines: negative after FMT; none at baseline; black lines: positive at baseline; none after FMT; orange lines: negative at baseline; none after FMT.

acquired using PURGE water suppression (25), PROJECT spin-echo, and J-resolved NMR sequences. NMR data from before and after FMT, before and after placebo, and after FMT and after placebo groups were compared using principal component analyses (KnowItAll Informatics, Metabolomics Edition v17.0, Bio-Rad) and partial least-squares discriminant analysis (MetaboAnalyst 4.0, McGill University) (26). Details can be found in the Supplemental Methods. 


\section{Systemic inflammation}

Serum IL-6 and serum LBP were analyzed using published ELISA techniques (Supplemental Methods).

Correlation networks. Correlation networks using published $\mathrm{R}$ techniques were ultimately created to determine linkages among gut microbiota, liver disease severity (MELD score), cognitive function, systemic inflammation, and serum and fecal BAs for the group receiving FMT (27). Pre-FMT baseline was compared with post-FMT day 20 assessments using R, and the specific correlation network differences were then highlighted. Cumulative distribution frequencies were compared among groups. We specifically focused on EncephalApp OffTime+OnTime performance, IL-6, LBP, and the 2 taxa for which the donor was selected, Lachnospiraceae and Ruminococcaceae.

Effect on outcomes. Finally, 3 of the FMT patients either developed an infection or were hospitalized and 6 of the placebo patients developed these outcomes. We analyzed the changes in microbial function in these patients compared to the remaining FMT and placebo recipients.

\section{Statistics}

We performed Wilcoxon matched-pairs analysis before/after FMT and before/after placebo as well as Mann-Whitney $U$ test for medians as appropriate. Multiple-comparison analyses were performed using Dunn's multiple-comparisons test as appropriate (Tables 1 and 2). Graphpad Prism and Minitab software was used for analysis and figures. Cytoscape was used to visualize correlation network differences, which were calculated using R software.

\section{Study approval}

This study was approved by the IRBs at Virginia Commonwealth University and McGuire VA Medical Center. All participants provided written, informed consent for the study.

\section{Author contributions}

JSB and NS obtained funding. NS, MLH, and MH were responsible for inflammatory analyses. HN, HT, GK, WMP, and PBH were involved in BA analysis. MBW, AF, MF, HL, SCM, PP, MSS, JSB, CA, RKS, VL, RTS, AJS, and EAG were involved in the clinical trial and sample collection and processing. IJC, SDTR, LA, RW, RAA, and ALG were involved in untargeted metabolomics, while MS and PMG were involved in microbiota analysis and bioinformatics.

\section{Acknowledgments}

This study was supported by a grant from the National Center for Advancing Translational Sciences/NIH (R21TR002024 to JSB and NS) and a VA Merit Review Grant (2I0CX001076 to JSB). SDTR is grateful to the United Kingdom National Institute for Health Research Biomedical Facility at Imperial College London for infrastructure support. The NMR Facility of the Centre for Biomolecular Spectroscopy was funded by British Heart Foundation, Wellcome Trust, and King's College London.

Address correspondence to: Jasmohan S. Bajaj, Division of Gastroenterology, Hepatology and Nutrition, Virginia Commonwealth University and McGuire VA Medical Center, 1201 Broad Rock Boulevard, Richmond, Virginia 23249, USA. Phone: 804.675.5802; Email: jasmohan.bajaj@vcuhealth.org.

1. Vilstrup H, et al. Hepatic encephalopathy in chronic liver disease: 2014 Practice Guideline by the American Association for the Study of Liver Diseases and the European Association for the Study of the Liver. Hepatology. 2014;60(2):715-735.

2. Acharya C, Bajaj JS. Altered microbiome in patients with cirrhosis and complications. Clin Gastroenterol Hepatol. 2019;17(2):307-321

3. Bajaj JS, et al. Fecal microbiota transplant from a rational stool donor improves hepatic encephalopathy: A randomized clinical trial. Hepatology. 2017;66(6):1727-1738.

4. Bajaj JS, et al. Fecal microbial transplant capsules are safe in hepatic encephalopathy: A phase 1, randomized, placebo-controlled trial. Hepatology. 2019;70(5):1690-1703.

5. Ridlon JM, Harris SC, Bhowmik S, Kang DJ, Hylemon PB. Consequences of bile salt biotransformations by intestinal bacteria. Gut Microbes. 2016;7(1):22-39.

6. Hatton G, Shawcross DL. Is treating the gut microbiome the key to achieving better outcomes in cirrhosis? Expert Rev Gastroenterol Hepatol. 2019;13(1):1-2.

7. Acharya C, Bajaj JS. Current management of hepatic encephalopathy. Am J Gastroenterol. 2018;113(11):1600-1612.

8. Bajaj JS, et al. Antibiotic-associated disruption of microbiota composition and function in cirrhosis is restored by fecal trans- 
plant. Hepatology. 2018;68(4):1549-1558

9. Kakiyama G, et al. Modulation of the fecal bile acid profile by gut microbiota in cirrhosis. J Hepatol. 2013;58 (5):949-955.

10. Weingarden AR, et al. Microbiota transplantation restores normal fecal bile acid composition in recurrent Clostridium difficile infection. Am J Physiol Gastrointest Liver Physiol. 2014;306(4):G310-G319.

11. Hofmann AF, Eckmann L. How bile acids confer gut mucosal protection against bacteria. Proc Natl Acad Sci USA. 2006;103(12):4333-4334.

12. Kang JD, et al. Bile acid $7 \alpha$-dehydroxylating gut bacteria secrete antibiotics that inhibit clostridium difficile: Role of secondary bile acids. Cell Chem Biol. 2019;26 (1):27-34.e4.

13. Shawcross DL. Is it time to target gut dysbiosis and immune dysfunction in the therapy of hepatic encephalopathy? Expert Rev Gastroenterol Hepatol. 2015;9(5):539-542.

14. Liu R, et al. Neuroinflammation in murine cirrhosis is dependent on the gut microbiome and is attenuated by fecal transplant [published online ahead of print June 20, 2019]. Hepatology. https://doi.org/10.1002/hep.30827.

15. Grander C, et al. Recovery of ethanol-induced Akkermansia muciniphila depletion ameliorates alcoholic liver disease. Gut 2018;67 (5):891-901.

16. Wu W, et al. Protective effect of akkermansia muciniphila against immune-mediated liver injury in a mouse model. Front Microbiol. 2017;8:1804

17. Belzer C, de Vos WM. Microbes inside--from diversity to function: the case of Akkermansia. ISME J. 2012;6(8):1449-1458.

18. Llorente C, et al. Gastric acid suppression promotes alcoholic liver disease by inducing overgrowth of intestinal Enterococcus Nat Commun. 2017;8(1):837.

19. Bajaj JS, et al. Modulation of the metabiome by rifaximin in patients with cirrhosis and minimal hepatic encephalopathy. PLoS One. 2013;8(4):e60042

20. DeFilipp Z, et al. Drug-Resistant E. coli Bacteremia Transmitted by Fecal Microbiota Transplant. N Engl J Med. 2019;381(21):2043-2050.

21. Kassam Z, et al. Donor Screening for Fecal Microbiota Transplantation. N Engl J Med. 2019;381(21):2070-2072.

22. Kakiyama G, et al. A simple and accurate HPLC method for fecal bile acid profile in healthy and cirrhotic subjects: validation by GC-MS and LC-MS. J Lipid Res. 2014;55(5):978-990.

23. Langille MG, et al. Predictive functional profiling of microbial communities using $16 \mathrm{~S}$ rRNA marker gene sequences. Nature biotechnology. 2013;31(9):814-821.

24. Dona AC, et al. Precision high-throughput proton NMR spectroscopy of human urine, serum, and plasma for large-scale metabolic phenotyping. Anal Chem. 2014;86(19):9887-9894.

25. Le Guennec A, Tayyari F, Edison AS. Alternatives to Nuclear Overhauser Enhancement Spectroscopy Presat and Carr-PurcellMeiboom-Gill Presat for NMR-Based Metabolomics. Anal Chem. 2017;89(17):8582-8588.

26. Chong J, et al. MetaboAnalyst 4.0: towards more transparent and integrative metabolomics analysis. Nucleic Acids Res. 2018;46 (W1):W486-W494.

27. Naqvi A, Rangwala H, Keshavarzian A, Gillevet P. Network-based modeling of the human gut microbiome. Chem Biodivers. 2010;7(5):1040-1050. 